\title{
Exploration of day-to-day route choice models by a virtual experiment
}

\author{
Hongbo Ye ${ }^{a}$, Feng Xiao ${ }^{\text {b, }}$, Hai Yang ${ }^{c}$ \\ a Institute for Transport Studies, University of Leeds, United Kingdom \\ ${ }^{b}$ School of Business Administration, Southwestern University of Finance and Economics, PR China \\ ${ }^{c}$ Department of Civil and Environmental Engineering, The Hong Kong University of Science and Technology, Clear Water Bay, Kowloon, Hong \\ Kong, PR China
}

\begin{abstract}
This paper examines existing day-to-day models based on a virtual day-to-day route choice experiment using the latest mobile internet techniques. With the realized day-to-day path flows and path travel times in the experiment, we calibrate several welldesigned path-based day-to-day models who take the Wardrop's user equilibrium as (part of) their stationary states. The nonlinear effects of path flows and path time differences on the path swapping are then investigated. Participants' path preferences, time-varying sensitivity and learning behavior in the day-to-day process are also examined. The prediction power of various models with various settings (nonlinear effects, time-varying sensitivity, and learning) is compared. Assumption of rational behavior adjustment process in Yang and Zhang (2009) is further verified. Finally, evolutions of different Lyapunov functions used in the literature are plotted and no obvious diversity is observed.
\end{abstract}

(C) 2017 The Authors. Elsevier B.V. All rights reserved.

Peer-review under responsibility of the scientific committee of the 22nd International Symposium on Transportation and Traffic Theory.

Keywords: Day-to-day flow dynamics; virtual route choice experiment; regression analysis; model calibration and comparison.

\section{Introduction and literature review}

There has been a substantial stream of development of day-to-day dynamic models to look into the variation of traffic flows from epoch to epoch (Cascetta, 1989; Watling and Cantarella, 2015). It is believed that travelers'

\footnotetext{
* Corresponding author.

E-mail address: xiaofeng@swufe.edu.cn
} 
historical traffic experience, as well as their prediction on future traffic conditions, would influence their trip decisions from day to day. Prediction of the traffic condition in the future epoch (e.g., traffic volume of the morning peak on working day) can help the transportation agencies arrange appropriate management and control strategies ahead of time. It is especially useful when the network structure changes (Guo and Liu, 2011; He and Liu, 2012). In general, two types of trip decision, i.e., route choice and departure time choice, are considered in the day-to-day study context. The focus of this paper is solely on route choice. Readers interested day-to-day departure time choices can refer to some pioneering works by Hu and Mahmassani (1997), Mahmassani (1990), Mahmassani and Chang (1986), Mahmassani et al., (1986), and recently by Xiao and Lo (2016), just to name a few.

Starting from the pioneer work by Smith (1984) and Horowitz (1984), the day-to-day route choice models are to study how the aggregate traffic flow changes based on current/historical flows and travel costs. The day-to-day model is a deterministic-process model if it is formulated as ordinary differential equations or difference equations, and their steady states can be different kinds of user equilibrium (UE), including deterministic UE (DUE, i.e. Wardrop's UE), stochastic UE (Cantarella and Cascetta, 1995; Smith and Watling, 2016), and boundedly rational UE (Di et al., 2015; Guo and Liu, 2011; Mahmassani and Chang, 1987; Ye and Yang, 2016). The stochastic-process models, on the other hand, formulate flow dynamics as stochastic processes, and the steady state is the equilibrium probability distribution (Cascetta, 1989; Cascetta and Cantarella; 1991; Davis and Nihan, 1993; Hazelton, 2002; Hazelton and Parry, 2016; Hazelton and Watling, 2004; Parry and Hazelton, 2013; Watling and Cantarella, 2015).

The interaction between day-to-day dynamic route flows and other components of the transportation system is widely studied in the analytical way, which includes the traffic information system (Bifulco et al., 2016; Cantarella, 2013; Cho and Hwang, 2005; Friesz et al., 1994), fixed or responsive signal control strategies (Cantarella et al., 2012; Huang et al., 2016; Liu and Smith, 2015; Smith et al., 2015; Smith and Mounce, 2011; Xiao and Lo, 2015), congestion pricing (Friesz et al., 2004; Farokhi and Johansson, 2015; Guo, 2013; Guo et al., 2016; Tan et al., 2015; Wang et al., 2015; Xu et al., 2016; Yang, 2007; Yang and Szeto, 2006; Yang et al., 2007; Ye et al., 2015), and tradable credit scheme (Ye and Yang, 2013). Day-to-day dynamics in other travel modes are also studied, such as in railway (Wu et al., 2013) and transit (Bar-Yosef et al., 2013; Cantarella et al., 2015; Li and Yang, 2016).

Besides theoretical development, the route choice based day-to-day dynamics is also studied through simulation and laboratory experiments. Most of these studies were concerned about how travelers' route choices are affected by various factors such as information, experience, risk, uncertainty, personality factors, as well as various transportation system components mentioned above (Avineri and Prashker, 2005, 2006; Ben-Elia et al., 2008, 2013; Hu and Mahmassani, 1997; Lotan, 1997; Lu et al., 2011; Mahmassani and Herman, 1990; Mahmassani and Stephan, 1988; Rapoport et al., 2014; Srinivasan and Mahmassani, 2003; Yang et al., 1993). The laboratory experiments were also used to test the static UE theories such as Braess Paradox and Downs-Thomson Paradox (Dechenaux et al., 2014; Morgan et al., 2009; Rapoport et al., 2009).

Our paper focuses on another interesting question that has not yet received sufficient attention in the research community: are the various route choice based day-to-day models proposed so far good enough to reflect the reallife situation, and if yes, what are their relative good performances? Regarding this question, some early and recent empirical studies are conducted by Avineri and Prashker (2005), He and Liu (2012), Mahmassani and Jou (2000), Meneguzzer and Olivieri (2013), Rapoport et al., (2014), just to name a few. In contrast, in this study we focus on a specific group of DUE-based day-to-day route choice models, which all have nice stability and convergence property but are not empirically studied yet. And to answer our question, we conducted a virtual route choice 
experiment and collected the participants' day-to-day route choice data using the latest mobile internet techniques. With the experimental data the existing day-to-day models are studied from the following aspects. First, the existing path-based day-to-day models in the literature are calibrated. Second, the nonlinear effects of path flows and path time differences on route switching are investigated. Third, participants' preferences to different paths, variation of their sensitivity over time, and their learning behavior are examined. Fourth, the assumption of "rational behavior adjustment process" is verified. Fifth, the prediction power of various day-to-day models is compared. Finally, various forms of Lyapunov functions used for stability analysis in the literature are examined.

The rest of this paper is organized as follows. Section 2 introduces the settings and processes of the virtual route choice experiment. Section 3 provides the findings from the quantitative analyses on the data. Section 4 draws the conclusions and discusses possible future directions.

\section{Introduction of the virtual route choice experiment}

To mimic travelers' decision-making processes from epoch to epoch in real life, the traditional laboratory or virtual experiments usually involved a relatively small number of participants and/or required the participants to repeatedly make decisions within short periods of time. In order to mimic the real world better, we managed to involve a larger number of participants and allow longer periods for decision-making, with the help of the social networking app Wechat. The network in Figure 1 was used, and the link travel times were calculated as $t_{a}\left(v_{a}\right)=t_{a}^{0}\left[1+0.15\left(v_{a} / Y_{a}\right)^{4}\right]$, with parameters given in Table 1 , where $v_{a}, t_{a}^{0}$ and $Y_{a}$ are respectively the flow, free flow time and capacity of link $a$. In our experiment, 268 participants took part for 26 rounds, where each round corresponded to a true calendar day. Most of the participants were students of the Southwest Jiaotong University in China. On the first day, the route map and the free flow times on the three paths were provided to the participants at 8:00 a.m. The participants had the entire daytime to determine which path to travel and were asked to submit their decisions before 9:00 p.m. When all the route choices were submitted, the path travel times would be calculated based on the predetermined travel time functions. Notably, the travel time functions were unknown to the participants. The participants choosing the shortest path(s) would then be the winners of that day and immediately get the monetary reward. The reward given to each winner was random, but in total equal to the number of winners multiplied by one Chinese Yuan per winner. On the second day and afterwards, both the route map and the path travel times on the previous day were provided (in minute and rounded to one decimal place) to the participants at 8:00 a.m. They would then make and submit decisions before 9:00 p.m.; the travel time would be calculated at night and the winners would be rewarded. The process proceeded until terminated by us.

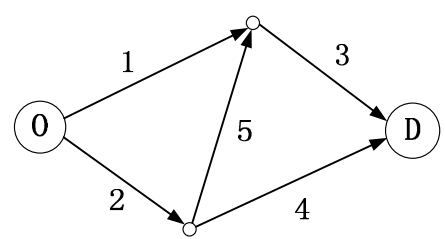

Figure 1. Network structure and paths.



Path 3 
Table 1. Link characteristics

\begin{tabular}{llllll}
\hline Link no. $(a)$ & 1 & 2 & 3 & 4 & 5 \\
Free flow time $\left(t_{a}^{0}\right)(\mathrm{min})$ & 25 & 10 & 5 & 20 & 15 \\
Capacity $\left(Y_{a}\right)$ & 40 & 80 & 80 & 40 & 40 \\
\hline
\end{tabular}

In order to win the reward, the participants would have incentive to choose the shortest path, which fits their behavior into the assumption of DUE. Therefore, our analyses in this paper will only focus on those day-to-day models whose equilibrium states are DUE. With the parameters given in Table 1, we can calculate a unique equilibrium path flow pattern of [89, 89, 89], with an identical path travel time of $142 \mathrm{~min}$. After plotting the observed day-to-day path flows and path travel times in Figure 2, we can find that, as the experiment proceeded, the fluctuation of path flows and path times became smaller and smaller, and on the $26^{\text {th }}$ round/day the network state was close to the equilibrium so we terminated the experiment. Furthermore, the average path travel time fluctuated even less and was very close to the equilibrium path time even at the early stage of the experiment.

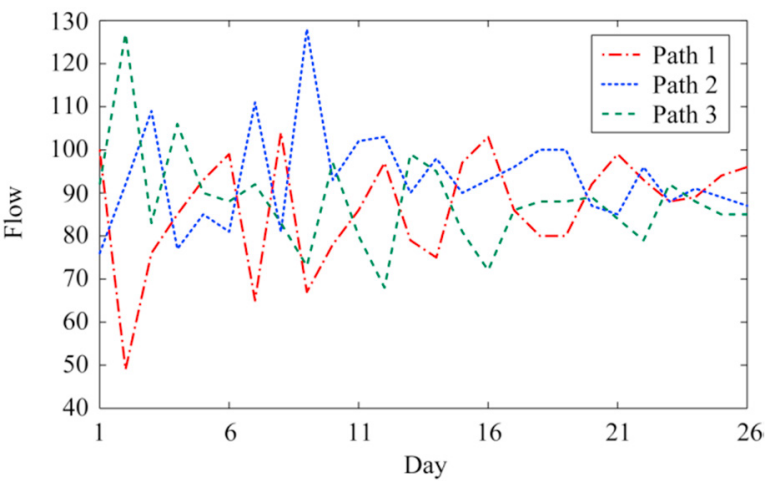

(a)

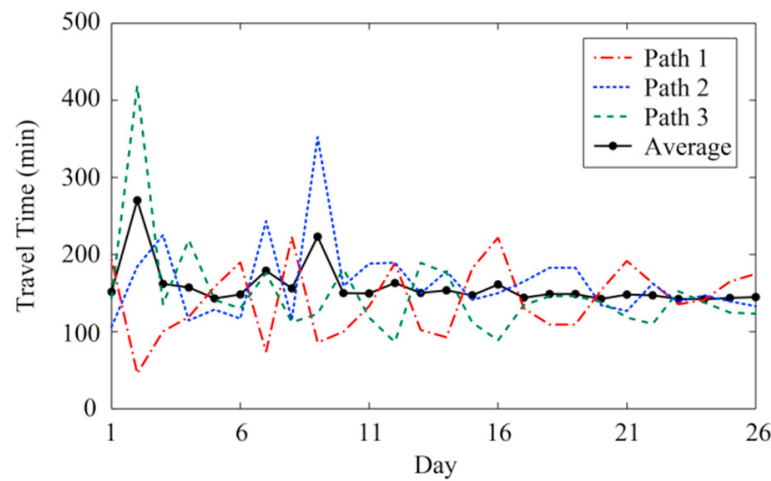

(b)

Figure 2. The day-to-day evolution of (a) path flows and (b) path travel times.

\section{Data analyses}

This section will be devoted to exploring the existing day-to-day models, by calibrating the parameters of these models based on the collected data. For the subsequent analyses, denote by $d=268$ the fixed origin-destination demand, $A=\{1,2,3,4,5\}$ the link set, and $R=\{1,2,3\}$ the path set. Let $f_{r}^{(n)}$ and $c_{r}^{(n)}$ respectively be the flow and actual travel time on path $r \in R$ on day $n, n=1,2, \ldots, 26$. Define $f^{(n)}=\left(f_{r}^{(n)}, r \in R\right)^{\mathrm{T}}$ and $c^{(n)}=\left(c_{r}^{(n)}, r \in R\right)^{\mathrm{T}}$ as the column vectors of path flows and path travel times, where " $\mathrm{T}$ " stands for the transpose operation.

The focus in this section will be on the first-order day-to-day models, in which the path flows on day $n+1$ are uniquely determined by the flows and travel times on day $n$. Denoting $g_{r s}^{(n+1)}$ as the flow swapping rate from path $r$ to path $s$ on day $n+1$, the general first-order day-to-day flow dynamics can be expressed as

$$
g_{r s}^{(n+1)}=\alpha \phi_{r s}^{(n+1)}\left(f^{(n)}, c^{(n)}\right), \alpha>0
$$


where $\phi_{r s}^{(n+1)}\left(f^{(n)}, c^{(n)}\right)$ is a function specifying how $f^{(n)}$ and $c^{(n)}$ determine the flow changing rate from path $r$ to path $s$, and normally it satisfies $\phi_{r s}^{(n+1)}=-\phi_{s r}^{(n+1)}$. The first-order day-to-day models investigated in this paper include the proportional-switch adjustment process (PSAP) in Smith (1984),

$$
\phi_{r s}^{(n+1)}=f_{r}^{(n)}\left[c_{r}^{(n)}-c_{s}^{(n)}\right]_{+}-f_{s}^{(n)}\left[c_{s}^{(n)}-c_{r}^{(n)}\right]_{+}
$$

where $[x]_{+}=\max (x, 0)$; the first-in-first-out (FIFO) dynamics in Jin (2007),

$$
\phi_{r s}^{(n+1)}=f_{r}^{(n)} f_{s}^{(n)}\left(c_{r}^{(n)}-c_{s}^{(n)}\right)
$$

the recent one in Xiao, Yang and Ye (2016), which is called as XYY dynamics afterwards,

$$
\phi_{r s}^{(n+1)}=c_{r}^{(n)}-c_{s}^{(n)}
$$

the evolutionary traffic flow dynamics (ETFD) in Yang (2005),

$$
\phi_{r s}^{(n+1)}=f_{r}^{(n)}\left[\bar{c}^{(n)}-c_{s}^{(n)}\right]_{+}-f_{s}^{(n)}\left[\bar{c}^{(n)}-c_{r}^{(n)}\right]_{+}
$$

where $\bar{c}^{(n)}=\sum_{r \in R} f_{r}^{(n)} c_{r}^{(n)} / d$ is the average travel time on day $n$, and the simplex gravity flow dynamics (SGFD) in Smith (1983),

$$
\phi_{r s}^{(n+1)}=\frac{f_{r}^{(n)}\left[\bar{c}^{(n)}-c_{s}^{(n)}\right]_{+}-f_{s}^{(n)}\left[\bar{c}^{(n)}-c_{r}^{(n)}\right]_{+}}{\sum_{s \in R}\left[\bar{c}^{(n)}-c_{s}^{(n)}\right]_{+}}
$$

The network tatonnement process (NTP) (Friesz et al., 1994) and the projected dynamic system (PDS) (Zhang and Nagurney, 1996; Nagurney and Zhang, 1997) are not investigated here for two reasons: first, they will degenerate to XYY dynamics under mild conditions (Xiao et al., 2016); second, their parameters cannot be estimated by the regression method used in this paper. The parameter $\alpha$ in Eq. (1) can be calibrated by both simulation and regression, under different forms of $\phi_{r s}^{(n+1)}$ in Eqs. (2)-(6).

\subsection{Simulation-based calibration}

The simulation-based method is to find the values of the parameters under which the simulated evolution process can fit best the observed flow swapping rates (in the sense of minimizing the sum of squared error between simulated and observed flow swapping rates). As shown in Figure 3, none of these five models can produce a fluctuation pattern close to the observation, and the simulated trajectories of PSAP, FIFO and XYY almost overlap with each other. 


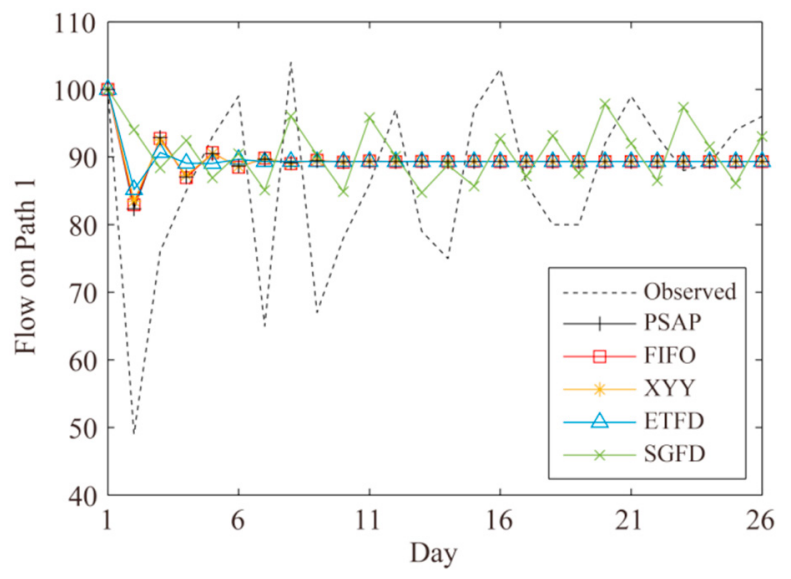

(a)



(b)

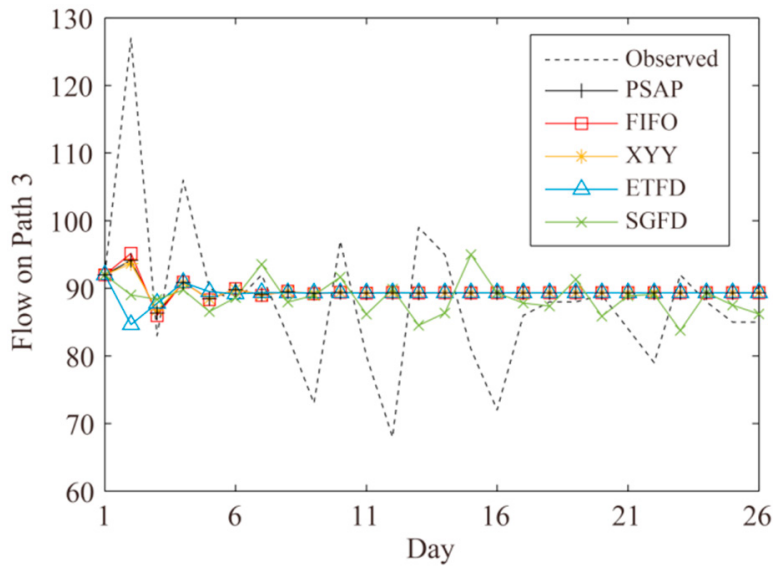

(c)

Figure 3. Best-fitted trajectories based on simulation: (a) Path 1; (b) Path 2; (c) Path 3.

\subsection{Regression-based calibration}

The simulation-based calibration shows the difficulty in reproducing the day-to-day flow pattern by one particular model with only one parameter. Therefore, we turn to a relaxed problem: given the path flows and costs of a particular day, how accurately can we predict the flows on the next day? For this problem, the regression-based calibration can be used. The regression analyses are conducted with the help of the built-in function regstats in Matlab R2016a. To clarify, $\hat{\alpha}$ denotes the calibrated value of parameter $\alpha$; the $p$-value associated with $\hat{\alpha}$ is obtained on a two-side test and indicates that the null hypothesis $(\alpha=0)$ is rejected at a significance level higher than this $p$-value. The heteroscedasticity is tested by the White test. The first-order autocorrelation is tested by the Ljung-Box Q test (or Q test for short), and the higher-order autocorrelations are not tested. The $p$-value of the White test ( $\mathrm{Q}$ test) indicates that the null hypothesis of homoscedasticity (autocorrelation-free) is rejected at a significance level higher than this $p$-value. In this study, we consider a significance level of $5 \%$ for both heteroscedasticity and autocorrelation, so they might need to be dealt with if the associated $p$-value is smaller than 0.05 . 


\subsubsection{The five original day-to-day models}

For the convenience of model comparison, define the day set $N=\{2,3, \cdots, 25\}$ throughout the whole Subsection 3.2. We begin the analyses with the five day-to-day models in Eqs. (2)-(6). The regression is based on the following formulation,

$$
g_{r s}^{(n+1)}=\alpha \phi_{r s}^{(n+1)}+\varepsilon_{r s}^{(n+1)}, n \in N,(r, s)=(1,2),(1,3),(2,3)
$$

where $\varepsilon_{r s}^{(n+1)}$ is the random error, and $\phi_{r s}^{(n+1)}$ is calculated by substituting the observed $f^{(n)}$ and user-informed $c^{(n)}$ into Eqs. (2)-(6). Notably, considering $g_{r s}^{(n+1)}=-g_{s r}^{(n+1)}$ and $\phi_{r s}^{(n+1)}=-\phi_{s r}^{(n+1)}$, only three path pairs are considered in the regression, and the intercept is excluded. The plots of $g_{r s}^{(n+1)}$ against $\phi_{r s}^{(n+1)}$ (Figure 4) show an origin-centric pattern and positive correlation between them.
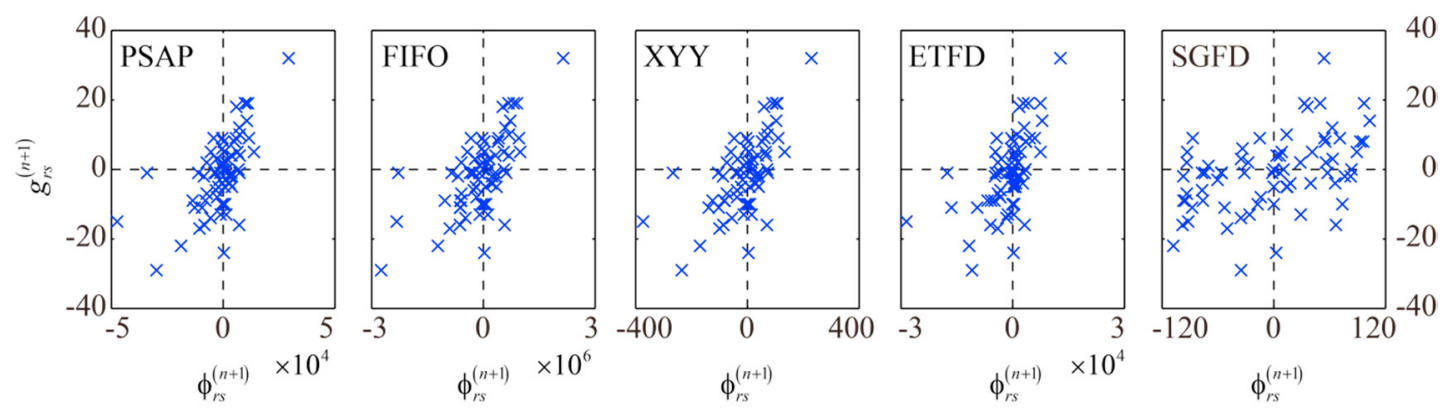

Figure 4. Plots of $g_{r s}^{(n+1)}$ against $\phi_{r s}^{(n+1)}$.

The result of ordinary least square (OLS) regression on Eq. (7) is listed in Table 2. The heteroscedasticity is detected in PSAP, XYY and ETFD. The autocorrelation is detected in SGFD. We will not try to correct for the autocorrelation of SGFD hereafter, until we discuss the learning behavior in Section 3.2.5.

Table 2. Calibration result of original models based on OLS

\begin{tabular}{|c|c|c|c|c|c|}
\hline & PSAP & FIFO & XYY & ETFD & SGFD \\
\hline$\alpha$ & $6.35 \mathrm{E}-4$ & $9.53 \mathrm{E}-6$ & $7.51 \mathrm{E}-2$ & $1.04 \mathrm{E}-3$ & $7.83 \mathrm{E}-2$ \\
\hline$p$-value & $2.16 \mathrm{E}-9$ & $1.66 \mathrm{E}-10$ & $7.51 \mathrm{E}-10$ & $5.91 \mathrm{E}-8$ & $6.32 \mathrm{E}-5$ \\
\hline White test ( $p$-value) & $0.007^{*}$ & 0.304 & $0.027^{*}$ & $0.036^{*}$ & 0.636 \\
\hline $\mathrm{Q}$ test ( $p$-value) & 0.328 & 0.588 & 0.370 & 0.092 & $0.019^{* *}$ \\
\hline
\end{tabular}

${ }^{*}$ Homoscedasticity rejected at $5 \%$ significance level

${ }^{* *}$ Autocorrelation-free rejected at 5\% significance level

The first way to tackle heteroscedasticity is to modify the model forms. Comparing the forms of ETFD and SGFD in Eqs. (5) and (6), SGFD shares the same term as ETFD but includes extra functions of travel times in the denominator. Enlightened by this, we simply modify PSAP, FIFO, XYY and ETFD by dividing the average travel time, which read

$$
\operatorname{PSAP}\left(\text { new): } \phi_{r s}^{(n+1)}=\frac{f_{r}^{(n)}\left[c_{r}^{(n)}-c_{s}^{(n)}\right]_{+}-f_{s}^{(n)}\left[c_{s}^{(n)}-c_{r}^{(n)}\right]_{+}}{\bar{c}^{(n)}}\right.
$$




$$
\begin{aligned}
& \text { FIFO (new): } \phi_{r s}^{(n+1)}=\frac{f_{r}^{(n)} f_{s}^{(n)}\left(c_{r}^{(n)}-c_{s}^{(n)}\right)}{\bar{c}^{(n)}} \\
& \text { XYY (new): } \phi_{r s}^{(n+1)}=\frac{c_{r}^{(n)}-c_{s}^{(n)}}{\bar{c}^{(n)}} \\
& \operatorname{ETFD~(new):~} \phi_{r s}^{(n+1)}=\frac{f_{r}^{(n)}\left[\bar{c}^{(n)}-c_{s}^{(n)}\right]_{+}-f_{s}^{(n)}\left[\bar{c}^{(n)}-c_{r}^{(n)}\right]_{+}}{\bar{c}^{(n)}}
\end{aligned}
$$

The calibration (Table 3 ) of these four new models gives statistically significant (at $1 \%$ level significance level) $\hat{\alpha}$, and the heteroscedasticity of PSAP, XYY and ETFD are corrected. Interestingly, the new ETFD model and the SGFD model have the same numerator but different denominators, while the former is autocorrelation-free but the latter is not. It is worth pointing out that, the model modification should not alter the properties of the original dayto-day model in terms of steady states and stability. Here under our modification, the steady states are unchanged,

\begin{tabular}{|c|c|c|c|c|}
\hline & PSAP (new) & FIFO (new) & XYY (new) & ETFD (new) \\
\hline$\hat{\alpha}$ & 0.132 & $1.84 \mathrm{E}-3$ & 14.5 & 0.221 \\
\hline$p$-value & $7.38 \mathrm{E}-10$ & $3.22 \mathrm{E}-10$ & $9.98 \mathrm{E}-10$ & $1.69 \mathrm{E}-08$ \\
\hline White test ( $p$-value) & 0.062 & 0.162 & 0.186 & 0.410 \\
\hline $\mathrm{Q}$ test ( $p$-value) & 0.508 & 0.655 & 0.456 & 0.181 \\
\hline
\end{tabular}
but the stability requires revisit.

Table 3. Calibration results of the modified models (OLS)

Bearing in mind the restrictions of doing model modification, we adopt the weighted least square (WLS) that does not need to change the model forms. In particular, the following WLS is used:

$$
\frac{g_{r s}^{(n+1)}}{\sqrt{\left|\phi_{r s}^{(n+1)}\right|}}=\alpha \frac{\phi_{r s}^{(n+1)}}{\sqrt{\left|\phi_{r s}^{(n+1)}\right|}}+\frac{\varepsilon_{r s}^{(n+1)}}{\sqrt{\left|\phi_{r s}^{(n+1)}\right|}}, n \in N,(r, s)=(1,2),(1,3),(2,3)
$$

Notably, the samples with $\phi_{r s}^{(n+1)}=0$ will be ruled out in the WLS regression. The result is given in Table 4 . Comparing with the OLS result in Table 3, the heteroscedasticity of PSAP, XYY and ETFD are corrected, although the autocorrelation of SGFD upholds. However, the significance level of $\hat{\alpha}$ is only 5\% in SGFD and even higher in other four models. And for the latter four models, the 95\% confidence interval (CI) shows tiny chance for $\alpha$ to be zero or even negative. Based on these observations, we will stick to OLS unless heteroscedasticity appears, and in the latter case, WLS will be applied instead.

Table 4. Calibration results of the original models (WLS)

\begin{tabular}{llllll}
\hline \multirow{2}{*}{ Model } & \multicolumn{3}{c}{$\alpha$} & $\begin{array}{l}\text { White test } \\
(p \text {-value })\end{array}$ & $\begin{array}{l}\text { Q test } \\
(p \text {-value })\end{array}$ \\
\cline { 2 - 4 } & $\hat{\alpha}$ & $p$-value & $95 \% \mathrm{CI}$ & 0.376 & 0.587 \\
\hline PSAP & $6.75 \mathrm{E}-4$ & 0.079 & {$[-0.074,1.3] \times 1 \mathrm{E}-3$} & 0.310 & 0.583 \\
FIFO & $8.97 \mathrm{E}-6$ & 0.062 & {$[-0.044,1.7] \times 1 \mathrm{E}-5$} & 0.310 & 0.503 \\
XYY & $7.12 \mathrm{E}-2$ & 0.054 & {$[-0.012,1.3] \times 1 \mathrm{E}-1$} & 0.250 & 0.197 \\
ETFD & $1.26 \mathrm{E}-3$ & 0.061 & {$[-0.054,2.4] \times 1 \mathrm{E}-3$} & 0.356 & $0.003^{*}$ \\
SGFD & $8.80 \mathrm{E}-2$ & 0.038 & {$[0.048,1.6] \times 1 \mathrm{E}-1$} & 0.137 & \\
\hline
\end{tabular}

${ }^{*}$ Autocorrelation-free rejected at 5\% significance level 


\subsubsection{Nonlinear effects of flows and cost differences on route switching}

Mounce and Carey (2011) suggested incorporating nonlinear effects in the original PSAP formulations. Following this idea, we define the following bivariate function $h(x, p)$,

$$
h(x, p)=\left\{\begin{array}{cc}
x^{p} & x>0 \\
0 & x=0 \\
-(-x)^{p} & x<0
\end{array}\right.
$$

and extend Eqs. (2)-(5) as follows,

$$
\begin{aligned}
& \text { PSAP: } \phi_{r s}^{(n+1)}(p, q)=h\left(f_{r}^{(n)}, p\right) h\left(\left[c_{r}^{(n)}-c_{s}^{(n)}\right]_{+}, q\right)-h\left(f_{s}^{(n)}, p\right) h\left(\left[c_{s}^{(n)}-c_{r}^{(n)}\right]_{+}, q\right) \\
& \text { FIFO: } \phi_{r s}^{(n+1)}(p, q)=h\left(f_{r}^{(n)}, p\right) h\left(f_{s}^{(n)}, p\right) h\left(c_{r}^{(n)}-c_{s}^{(n)}, q\right) \\
& \text { XYY: } \phi_{r s}^{(n+1)}(p, q)=h\left(c_{r}^{(n)}-c_{s}^{(n)}, q\right) \\
& \text { ETFD: } \phi_{r s}^{(n+1)}(p, q)=h\left(f_{r}^{(n)}, p\right) h\left(\left[\bar{c}^{(n)}-c_{s}^{(n)}\right]_{+}, q\right)-h\left(f_{s}^{(n)}, p\right) h\left(\left[\bar{c}^{(n)}-c_{r}^{(n)}\right]_{+}, q\right)
\end{aligned}
$$

where parameters $p$ and $q$ capture the degree of nonlinearity. Finding the best values of $p$ and $q$ in each model can be treated as a nonlinear regression problem

$$
\min _{p, q, \alpha} \sum_{n \in N} \sum_{(r, s)}\left[g_{r s}^{(n+1)}-\alpha \phi_{r s}^{(n+1)}(p, q)\right]^{2}
$$

The results (Table 5) prefer a slightly concave relation between cost differences and swapping rates for all four models, while the relations between flow and swapping rates are quite different: although they all suggest convex relations, PSAP suggests a decreasing one but FIFO and ETFD suggest an increasing one. The day-to-day models in Eqs. (14)-(17) with these optimal $p$ and $q$ values are then calibrated, and the results are given in Table 6 .

Table 5. Optimal parameter values for capturing nonlinear effects

\begin{tabular}{lllll}
\hline & PSAP & FIFO & XYY & ETFD \\
\hline$p$ & -0.69 & 1.20 & - & 3.12 \\
$q$ & 0.99 & 0.92 & 0.89 & 0.31 \\
\hline
\end{tabular}

Table 6. Calibration based on optimal $p$ and $q$ values (OLS)

\begin{tabular}{llllll}
\hline & & PSAP & FIFO & XYY & ETFD \\
\hline$\alpha$ & $\hat{\alpha}$ & 2.07 & $2.35 \mathrm{E}-6$ & 0.13 & $1.20 \mathrm{E}-6$ \\
& $p$-value & $5.73 \mathrm{E}-10$ & $1.42 \mathrm{E}-10$ & $6.00 \mathrm{E}-10$ & $1.21 \mathrm{E}-9$ \\
White test $(p$-value $)$ & 0.084 & 0.475 & 0.073 & 0.057 \\
Q test $(p$-value $)$ & 0.369 & 0.629 & 0.406 & 0.483 \\
\hline
\end{tabular}

\subsubsection{Path preferences}

Being curious about whether the paths are treated differently when participants were making route choices, we write

$$
g_{r s}^{(n+1)}=\gamma_{r s}+\alpha_{r s} \phi_{r s}^{(n+1)}+\varepsilon_{r s}^{(n+1)}, n \in N,(r, s)=(1,2),(1,3),(2,3)
$$

where $\gamma_{r s}$ represents participants' preference between paths $r$ and $s$, and $\alpha_{r s}$ is the path-specific sensitivity. The following WLS will be used if heteroscedasticity is detected (at the 5\% significance level) in OLS: 


$$
\frac{g_{r s}^{(n+1)}}{\sqrt{\left|\phi_{r s}^{(n+1)}\right|}}=\gamma_{r s} \frac{1}{\sqrt{\left|\phi_{r s}^{(n+1)}\right|}}+\alpha_{r s} \frac{\phi_{r s}^{(n+1)}}{\sqrt{\left|\phi_{r s}^{(n+1)}\right|}}+\frac{\varepsilon_{r s}^{(n+1)}}{\sqrt{\left|\phi_{r s}^{(n+1)}\right|}}, n \in N,(r, s)=(1,2),(1,3),(2,3)
$$

The result is given in Table 7. The $\hat{\gamma}_{r s}$ values show that Path 2 is most preferred; however, this is not evident according to the $p$-value and 95\% CI. Again, the autocorrelation is detected (at 5\% significance level) in SGFD between path pair $(2,3)$.

Table 7. Calibration results on the path preference

\begin{tabular}{|c|c|c|c|c|c|c|c|c|c|}
\hline \multirow{2}{*}{ Model } & \multirow{2}{*}{$(r, s)$} & \multicolumn{3}{|c|}{$\gamma_{r s}$} & \multicolumn{2}{|c|}{$\alpha_{r s}$} & \multirow{2}{*}{ Is OLS? } & \multirow{2}{*}{$\begin{array}{l}\text { White test } \\
\text { ( } p \text {-value) }\end{array}$} & \multirow{2}{*}{$\begin{array}{l}\mathrm{Q} \text { test } \\
\text { ( } p \text {-value) }\end{array}$} \\
\hline & & $\hat{\gamma}_{r s}$ & $p$-value & $95 \% \mathrm{CI}$ & $\hat{\alpha}_{r s}$ & $p$-value & & & \\
\hline \multirow{3}{*}{ PSAP } & $(1,2)$ & 0.06 & 0.927 & {$[-1.18,1.29]$} & 7.02E-4 & 0.0477 & \multirow[t]{3}{*}{ No } & 0.459 & 0.609 \\
\hline & $(1,3)$ & 0.13 & 0.934 & {$[-3.08,3.34]$} & $5.10 \mathrm{E}-4$ & 0.0009 & & 0.793 & 0.891 \\
\hline & $(2,3)$ & -2.69 & 0.160 & {$[-6.52,1.14]$} & $9.77 \mathrm{E}-4$ & 0.0000 & & 0.860 & 0.597 \\
\hline \multirow{3}{*}{ FIFO } & $(1,2)$ & 0.09 & 0.887 & {$[-1.21,1.39]$} & $9.46 \mathrm{E}-6$ & 0.0369 & \multirow[t]{3}{*}{ No } & 0.397 & 0.595 \\
\hline & $(1,3)$ & -0.12 & 0.935 & {$[-3.10,2.86]$} & $9.31 \mathrm{E}-6$ & 0.0002 & & 0.704 & 0.818 \\
\hline & $(2,3)$ & -2.32 & 0.233 & {$[-6.23,1.59]$} & $1.15 \mathrm{E}-5$ & 0.0001 & & 0.858 & 0.455 \\
\hline \multirow{3}{*}{ XYY } & $(1,2)$ & 0.03 & 0.956 & {$[-1.17,1.24]$} & $7.37 \mathrm{E}-2$ & 0.0360 & \multirow[t]{3}{*}{ No } & 0.353 & 0.583 \\
\hline & $(1,3)$ & 0.12 & 0.936 & {$[-2.94,3.18]$} & $6.25 \mathrm{E}-2$ & 0.0003 & & 0.751 & 0.962 \\
\hline & $(2,3)$ & -2.97 & 0.132 & {$[-6.90,0.95]$} & $1.16 \mathrm{E}-1$ & 0.0001 & & 0.927 & 0.550 \\
\hline \multirow{3}{*}{ ETFD } & $(1,2)$ & 1.12 & 0.554 & {$[-2.75,4.99]$} & $8.86 \mathrm{E}-4$ & 0.0080 & & 0.367 & 0.386 \\
\hline & $(1,3)$ & 0.63 & 0.691 & {$[-2.61,3.87]$} & $8.58 \mathrm{E}-4$ & 0.0008 & & 0.838 & 0.862 \\
\hline & $(2,3)$ & -3.36 & 0.090 & {$[-7.28,0.56]$} & $2.24 \mathrm{E}-3$ & 0.0000 & & 0.771 & 0.757 \\
\hline \multirow{3}{*}{ SGFD } & $(1,2)$ & 0.60 & 0.749 & {$[-3.23,4.44]$} & $7.94 \mathrm{E}-2$ & 0.0136 & & 0.136 & 0.084 \\
\hline & $(1,3)$ & -0.36 & 0.819 & {$[-3.56,2.84]$} & $8.50 \mathrm{E}-2$ & 0.0010 & & 0.212 & 0.886 \\
\hline & $(2,3)$ & -2.65 & 0.347 & {$[-8.37,3.06]$} & $8.83 \mathrm{E}-2$ & $0.1235^{*}$ & & 0.870 & $0.031^{* *}$ \\
\hline
\end{tabular}

${ }^{*}$ Null hypothesis $\alpha_{r \mathrm{r}}=0$ not rejected at $5 \%$ significance level

${ }^{* *}$ Autocorrelation-free rejected at 5\% significance level

\subsubsection{Time-varying parameters}

Horowitz (1984) assumed that travelers' sensitivity to the path time difference can change along the evolution process. Under this circumstance, the parameters calibrated from historical data may not work well for predicting the future traffic condition. To examine this effect, we set up a time window of 15 days, calibrate the day-to-day processes in Eqs. (2)-(6) with observations in this time window, and see how $\hat{\alpha}$ changes as the time window rolls forward. The time window is $M=\{m-14, m-13, \cdots, m\}$, and $m$ rolls from 16 to 25 . Again, WLS

$$
\frac{g_{r s}^{(n+1)}}{\bar{c}^{(n)}}=\alpha_{r s} \frac{\phi_{r s}^{(n+1)}}{\bar{c}^{(n)}}+\frac{\varepsilon_{r s}^{(n+1)}}{\bar{c}^{(n)}}, n \in M,(r, s)=(1,2),(1,3),(2,3)
$$

will be used if OLS rejects homoscedasticity at a 5\% significance level. Here the denominator is different from $\sqrt{\left|\phi_{r s}^{(n+1)}\right|}$ used in earlier subsections in order to get a statistically significant and heteroscedasticity-free result. For all regressions, we have $\alpha=0$ rejected at 5\% significance level and homoscedasticity not rejected at 5\% significance level. Unfortunately, autocorrelation-free is rejected at 5\% significance level in 4 out of 10 regressions for SGFD. The evolution of $\hat{\alpha}$ is demonstrated in Figure 5. As we can see, the SGFD model shows an obvious decreasing trend on $\hat{\alpha}$, while the trend is less obvious in the other four models. However, the trend in SGFD is problematic due to the existence of autocorrelation! 


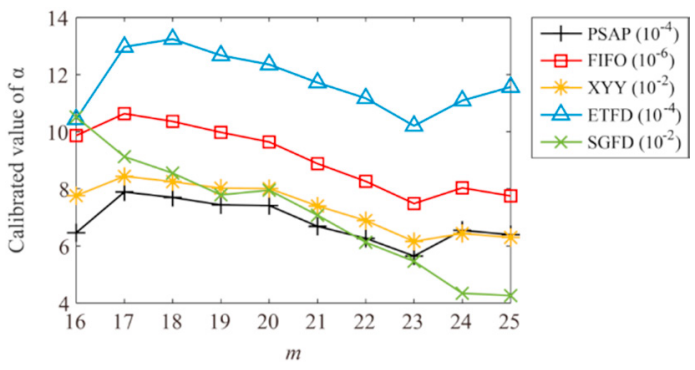

Figure 5. Evolution of $\hat{\alpha}$ with a rolling time window.

Following this trend, we assume a linear relation between parameter $\alpha$ and time/day $n$ as

$$
\alpha=\alpha(n)=\theta n+\mu
$$

Substitute it into Eq. (1) leads to

$$
g_{r s}^{(n+1)}=\theta \times(n+1) \phi_{r s}^{(n+1)}+\mu \times \phi_{r s}^{(n+1)}
$$

The following WLS is used if heteroscedasticity is detected (at $5 \%$ significance level) in OLS:

$$
\frac{g_{r s}^{(n+1)}}{\bar{c}^{(n)}}=\theta \times \frac{(n+1) \phi_{r s}^{(n+1)}}{\bar{c}^{(n)}}+\mu \times \frac{\phi_{r s}^{(n+1)}}{\bar{c}^{(n)}}
$$

The calibration result of Eq. (22) w.r.t. the five models in Eqs. (2)-(6) are given in Table 8. The $\hat{\theta}$ values indicate a decreasing trend of $\alpha$; however, it is not significant at 5\% level in all models except SFGD; however, not surprisingly, SGFD is not autocorrelation-free (at 5\% significance level). An interesting finding is that, comparing the formulations of ETFD and SFGD, the inclusion of denominator $\sum_{s \in R}\left[\bar{c}^{(n)}-c_{s}^{(n)}\right]_{+}$changes $\alpha$ from timeinvariant to time-varying. The explanation might be that, as the experiment proceeds, the system will evolve closer to the equilibrium, and thus the value of $\sum_{s \in R}\left[\bar{c}^{(n)}-c_{s}^{(n)}\right]_{+}$will gradually decrease. Such a decreasing trend would counteract the decreasing trend of $\alpha$, so the ETFD model shows no time-dependency. It is unclear why ETFD is

\begin{tabular}{|c|c|c|c|c|c|c|c|c|}
\hline \multirow{2}{*}{ Model } & \multirow{2}{*}{ OLS/WLS } & \multicolumn{3}{|c|}{$\theta$} & \multicolumn{2}{|c|}{$\mu$} & \multirow{2}{*}{$\begin{array}{l}\text { White test } \\
\text { ( } p \text {-value) }\end{array}$} & \multirow{2}{*}{$\begin{array}{l}\mathrm{Q} \text { test } \\
\text { ( } p \text {-value })\end{array}$} \\
\hline & & $\hat{\theta}$ & $p$-value & $95 \% \mathrm{CI}$ & $\hat{\mu}$ & $p$-value & & \\
\hline PSAP & WLS & $-1.13 \mathrm{E}-5$ & 0.595 & {$[-5.37,3.10] \times 1 \mathrm{E}-5$} & $8.04 \mathrm{E}-4$ & $8.30 \mathrm{E}-4$ & 0.416 & 0.477 \\
\hline FIFO & WLS & $-3.39 \mathrm{E}-7$ & 0.222 & {$[-8.89,2.10] \times 1 \mathrm{E}-7$} & $1.32 \mathrm{E}-5$ & $8.65 \mathrm{E}-5$ & 0.517 & 0.581 \\
\hline XYY & WLS & $-2.36 \mathrm{E}-3$ & 0.291 & {$[-6.78,2.07] \times 1 \mathrm{E}-3$} & 0.1019 & $1.82 \mathrm{E}-4$ & 0.518 & 0.451 \\
\hline ETFD & WLS & $-2.69 \mathrm{E}-8$ & 0.999 & {$[-7.74,7.74] \times 1 \mathrm{E}-5$} & 0.0012 & 7.72E-3 & 0.260 & 0.220 \\
\hline SGFD & OLS & $-8.00 \mathrm{E}-3$ & $0.004^{*}$ & {$[-1.34,-0.26] \times 1 \mathrm{E}-2$} & 0.1932 & $2.50 \mathrm{E}-5$ & 0.437 & $0.022^{* *}$ \\
\hline
\end{tabular}
autocorrelation-free but SGFD is not.

Table 8. Calibration results with time varying parameter

${ }^{*}$ Null hypothesis $\theta=0$ rejected at $1 \%$ significance level

${ }^{* *}$ Autocorrelation-free rejected at $5 \%$ significance level

To confirm our conjecture on the cause of difference between ETFD and SGFD, we redo the regression of Eq. (22) with $\phi_{r s}^{(n+1)}$ from Eqs. (8)-(11). The results in Table 8 suggest a time-dependent $\alpha$ for the new FIFO model. What we can find in this subsection is that, the assumption of time-varying parameters is actually associated with the model. 
Table 9. Calibration results on the time-varying parameter in new models (OLS only)

\begin{tabular}{|c|c|c|c|c|c|c|c|}
\hline \multirow{2}{*}{ Model } & \multicolumn{3}{|c|}{$\theta$} & \multicolumn{2}{|c|}{$\mu$} & \multirow{2}{*}{$\begin{array}{l}\text { White test } \\
\text { ( } p \text {-value) }\end{array}$} & \multirow{2}{*}{$\begin{array}{l}\mathrm{Q} \text { test } \\
\text { ( } p \text {-value) }\end{array}$} \\
\hline & $\hat{\theta}$ & $p$-value & $95 \% \mathrm{CI}$ & $\hat{\mu}$ & $p$-value & & \\
\hline PSAP (new) & $-4.61 \mathrm{E}-3$ & 0.173 & {$[-1.13,0.21] \times 1 \mathrm{E}-2$} & 0.18 & $7.88 \mathrm{E}-6$ & $0.029^{* *}$ & 0.413 \\
\hline FIFO (new) & $-9.44 \mathrm{E}-5$ & $0.032^{*}$ & {$[-1.80,-0.08] \times 1 \mathrm{E}-4$} & $2.78 \mathrm{E}-3$ & $3.65 \mathrm{E}-7$ & 0.076 & 0.509 \\
\hline XYY (new) & -0.68 & 0.057 & {$[-1.38,0.02]$} & 21.39 & $1.63 \mathrm{E}-6$ & 0.097 & 0.378 \\
\hline ETFD (new) & $-4.97 \mathrm{E}-3$ & 0.429 & {$[-1.74,0.75] \times 1 \mathrm{E}-2$} & 0.27 & $2.02 \mathrm{E}-4$ & 0.078 & 0.155 \\
\hline
\end{tabular}

${ }^{*}$ Null hypothesis $\theta=0$ rejected at $5 \%$ significance level

${ }^{* *}$ Homoscedasticity rejected at $5 \%$ significance level

\subsubsection{User learning in the day-to-day process}

Previous research also tried to explicitly model how travelers predict the future travel costs based on their experience, usually by an exponential smoothing rule (Bie and Lo, 2010; Cascetta and Cantarella, 1993; Cantarella and Cascetta, 1995; Horowitz, 1984; Watling, 1999; Xiao et al., 2016; Ye and Yang, 2013). In Xiao et al. (2016), the XYY model in Eq. (4) is modified by replacing the experienced time $c_{r}^{(n)}$ with the perceived/predicted time $C_{r}^{(n+1)}$ on path $r \in R$ on day $n+1$, i.e.,

$$
g_{r s}^{(n+1)}=\alpha\left(C_{r}^{(n+1)}-C_{s}^{(n+1)}\right), \alpha>0
$$

and $C_{r}^{(n+1)}$ is updated through the following exponential smoothing rule,

$$
C_{r}^{(n+1)}=\beta c_{r}^{(n)}+(1-\beta) C_{r}^{(n)}, 0<\beta \leq 1
$$

Substituting Eq. (25) into Eq. (24) yields

$$
g_{r s}^{(n+1)}=(1-\beta) g_{r s}^{(n)}+\alpha \beta\left(c_{r}^{(n)}-c_{s}^{(n)}\right), \alpha>0,0<\beta \leq 1
$$

Unrigorously, Eq. (26) can be easily extended to other day-to-day models, which leads to a general day-to-day model with learning,

$$
g_{r s}^{(n+1)}=(1-\beta) g_{r s}^{(n)}+\alpha \beta \phi_{r s}^{(n+1)}, \alpha>0,0<\beta \leq 1
$$

where $\phi_{r s}^{(n)}$ takes those forms in Eqs. (2)-(6). Calibration result of this learning model is given in Table 10, where the WLS is based on

$$
\frac{g_{r s}^{(n+1)}}{\sqrt{\phi_{r s}^{(n+1)}}}=(1-\beta) \frac{g_{r s}^{(n)}}{\sqrt{\phi_{r s}^{(n+1)}}}+\alpha \beta \frac{\phi_{r s}^{(n+1)}}{\sqrt{\phi_{r s}^{(n+1)}}}+\frac{\varepsilon_{r s}^{(n+1)}}{\sqrt{\phi_{r s}^{(n+1)}}}
$$

Again, we have some interesting observations.

(1) The assumption of learning is not supported in PSAP, FIFO, XYY or ETFD. If we only look at the value of $1-\beta$, all these four methods suggest $1-\beta<0$, or equally $\beta>1$. In particular, for the XYY model, according to Eq. (25), a $\beta>1$ obviously violates the widely-used assumption of $0<\beta \leq 1$ in the literature. However, is this completely impossible? By rewriting Eq. (25) into the following form,

$$
C_{r}^{(n+1)}=C_{r}^{(n)}+\beta\left(c_{r}^{(n)}-C_{r}^{(n)}\right)
$$

the learning process can now be interpreted as that, travelers will correct their previous perception/prediction by adding or subtracting a proportion $\beta$ of the difference between actual and perceived/predicted travel times. As a result, the perceived/predicted time would increase if $c_{r}^{(n)}>C_{r}^{(n)}$ and decrease otherwise. From a practical point of view, both $0<\beta \leq 1$ and $\beta>1$ could happen in reality: with $0<\beta \leq 1$, travelers are relatively "conservative" 
when learning the travel times, and they are relatively "aggressive" if $\beta>1$.

(2) The SGFD model also suggests $1-\beta=-0.317$, which is statistically significant at $1 \%$ level. The $95 \%$ CI also shows that $1-\beta$ is quite likely to be negative, which reveals some learning-like pattern. However, different from the XYY model, such "learning" is not on travel cost but on $\phi_{r s}^{(n+1)}$.

(3) The SGFD is finally autocorrelation-free! Reminding the autocorrelation of SGFD in Table 2, such learning formulation in Eq. (27) is actually a standard form to correct for autocorrelation. Moreover, since the other four models in Table 2 are autocorrelation-free, it is not surprising that they show no learning behavior here. The difference between ETFD and SGFD again tells us that the learning behavior is also model-dependent.

Table 10. Calibration results for user learning models

\begin{tabular}{|c|c|c|c|c|c|c|c|c|}
\hline \multirow{2}{*}{ Model } & \multirow{2}{*}{ OLS/WLS } & \multicolumn{3}{|c|}{$1-\beta$} & \multicolumn{2}{|c|}{$\alpha \beta$} & \multirow{2}{*}{$\begin{array}{l}\text { White test } \\
\text { ( } p \text {-value) }\end{array}$} & \multirow{2}{*}{$\begin{array}{l}\mathrm{Q} \text { test } \\
\text { ( } p \text {-value })\end{array}$} \\
\hline & & $1-\beta$ & $p$-value & $95 \% \mathrm{CI}$ & $\alpha \beta$ & $p$-value & & \\
\hline PSAP & WLS & -0.086 & 0.379 & {$[-0.28,0.11]$} & $5.47 \mathrm{E}-4$ & 0.131 & 0.609 & 0.681 \\
\hline FIFO & OLS & -0.107 & 0.300 & {$[-0.31,0.10]$} & $8.55 \mathrm{E}-6$ & $9.39 \mathrm{E}-7$ & 0.309 & 0.736 \\
\hline XYY & OLS & -0.109 & 0.308 & {$[-0.32,0.10]$} & $6.67 \mathrm{E}-2$ & $4.35 \mathrm{E}-6$ & 0.122 & 0.498 \\
\hline ETFD & OLS & -0.185 & 0.090 & {$[-0.40,0.03]$} & $8.35 \mathrm{E}-4$ & $1.35 \mathrm{E}-4$ & 0.195 & 0.262 \\
\hline SGFD & OLS & -0.317 & $0.002^{*}$ & {$[-0.52,-0.12]$} & $5.42 \mathrm{E}-2$ & $5.65 \mathrm{E}-3$ & 0.806 & 0.420 \\
\hline
\end{tabular}

${ }^{*}$ Null hypothesis $1-\beta=0$ rejected at $1 \%$ significance level

Now we can go back to handle the autocorrelation reported earlier on SGFD (Table 7 and Table 8). The pathspecific SGFD with learning now reads

$$
g_{r s}^{(n+1)}=\gamma_{r s}+\left(1-\beta_{r s}\right) g_{r s}^{(n)}+\alpha_{r s} \beta_{r s} \phi_{r s}^{(n+1)}, n \in N,(r, s)=(1,2),(1,3),(2,3)
$$

and the OLS results are given in Table 11. The autocorrelation between path pair $(1,3)$ is corrected. No path preference is confirmed according to the statistics on $\gamma_{r s}$. The path switching on path pairs $(1,2)$ and $(1,3)$ shows no obvious learning behavior, but is positively correlated to $\phi_{r s}^{(n+1)}$, although there exists a small possibility that $\alpha_{12} \beta_{12}$ for path pair $(1,2)$ could be zero or negative. The learning-like pattern on path pair $(2,3)$ is possible, with $1-\beta_{23}<0$; however, it seems that its flow swapping is now not quite determined by $\phi_{12}^{(n+1)}$.

Table 11. Calibration of SGFD with user learning and path preference (OLS)

\begin{tabular}{lllllll}
\hline$(r, s)$ & parameter & calibrated & $p$-value & $95 \%$ CI & White test $(p$-value $)$ & Q test $(p$-value $)$ \\
\hline \multirow{3}{*}{$(1,2)$} & $\gamma_{r s}$ & 0.163 & 0.928 & {$[-3.52,3.84]$} & & \\
& $1-\beta_{r s}$ & -0.296 & 0.083 & {$[-0.631,0.040]$} & 0.176 & 0.326 \\
& $\alpha_{r s} \beta_{r s}$ & 0.061 & 0.056 & {$[-0.001,0.123]$} & & \\
\hline \multirow{2}{*}{$(1,3)$} & $\gamma_{r s}$ & -0.356 & 0.824 & {$[-3.63,2.92]$} & & 0.943 \\
& $1-\beta_{r s}$ & -0.014 & 0.935 & {$[-0.360,0.332]$} & 0.294 & \\
& $\alpha_{r s} \beta_{r s}$ & 0.084 & $0.003^{*}$ & {$[0.031,0.137]$} & & 0.392 \\
& $\gamma_{r s}$ & -1.597 & 0.523 & {$[-6.68,3.48]$} & & \\
\hline & $1-\beta_{r s}$ & -0.550 & $0.012^{* *}$ & {$[-0.965,-0.135]$} & 0.581 & \\
& $\alpha_{r s} \beta_{r s}$ & 0.012 & 0.838 & {$[-0.104,0.127]$} & &
\end{tabular}

${ }^{*}$ Null hypothesis $\alpha_{r s} \beta_{r s}=0$ rejected at $1 \%$ significance level

${ }^{* *}$ Null hypothesis $1-\beta_{r s}=0$ rejected at $2 \%$ significance level 
The SGFD with learning, time-varying $\alpha$ and constant $\beta$ writes

$$
g_{r s}^{(n+1)}=(1-\beta) g_{r s}^{(n)}+\theta \beta \times(n+1) \phi_{r s}^{(n+1)}+\mu \beta \phi_{r s}^{(n+1)}
$$

and the OLS result is given in Table 12. Not surprisingly, the autocorrelation is corrected, and the homoscedasticity persists. The learning-like behavior is possible, with $1-\beta<0 . \alpha$ is possible to decrease with time, since $\beta>1$ and $\theta \beta<0$.

Table 12. Calibration of SGFD with user learning and time-varying parameter (OLS)

\begin{tabular}{llllll}
\hline parameter & calibrated & $p$-value & $95 \% \mathrm{CI}$ & White test $(p$-value $)$ & $\mathrm{Q}$ test $(p$-value $)$ \\
\hline $1-\beta$ & -0.26 & 0.0113 & {$[-0.46,-0.06]$} & & \\
$\theta \beta$ & $-6.38 \mathrm{E}-3$ & 0.0204 & {$[-11.7,-1.0] \times 1 \mathrm{E}-3$} & 0.627 & 0.267 \\
$\mu \beta$ & 0.15 & 0.0012 & {$[0.06,0.24]$} & & \\
\hline
\end{tabular}

\subsection{Model comparison based on regression results}

To evaluate and compare the prediction power of all the day-to-day models we have investigated so far, the root mean square error (RMSE), defined as $R M S E=\sqrt{\sum_{n \in N} \sum_{(r, s)}\left(\hat{g}_{r s}^{(n+1)}-g_{r s}^{(n+1)}\right)^{2} /(3 \times 24)}$, is a standard indicator, where $\hat{g}_{r s}^{(n+1)}$ is the predicted flow swapping rate based on the calibrated parameter value(s). Since RMSE is not intuitive in explaining the accuracy of prediction, we further define indicator AE- $x(x=10$ or 20$)$ as the proportions of those samples which satisfy $\left|\hat{f}_{r}^{(n+1)}-f_{r}^{(n+1)}\right| \leq x$ in the total 72 samples, where $\hat{f}_{r}^{(n+1)}$ is the predicted path flow based on the calibrated parameter value(s).

The values of RMSE, AE-10 and AE-20 are calculated based on the calibrated parameter values in Section 3.2 and given in

Table 13. Before discussing them, we have to emphasize that our conclusion given below is very rough. The heteroscedasticity and autocorrelation may not be corrected in some cases, and sometimes the WLS replaced the OLS for tackling heterogeneity. However, different WLS forms are used in different places, the WLS does not necessarily have the same sample set as that of OLS, and the OLS minimizes the RMSE but the WLS does not. All of these affect the numbers listed in

Table 13. The comparisons are made below.

(1) Regarding RMSE, FIFO $>$ PSAP $>$ XYY $>$ ETFD $>$ SGFD (where "> means "better than"), but the gaps between the first three models are small. Regarding AE-10 and AE-20, FIFO PSAP XYY $>$ ETFD $>$ SGFD (where “ ” means "similar to").

(2) Altering the function form of the day-to-day models does not necessary improve the prediction power, compared with the original form.

(3) The WLS does not necessary improve the prediction power, compared with OLS.

(4) Compared with the original day-to-day models, the forms incorporating nonlinear effects unsurprisingly reduce the RMSE, and the AE-20 is also better off but the AE-10 is worse off.

(5) Considering the time-varying parameter or learning behavior ought to reduce the RMSE value (but not necessarily AE-10 and AE-20) than without considering them; however, the WLS counteracts such improvement, and even when OLS is used, such improvement is very mild. 
Table 13. Performance of different models in prediction

\begin{tabular}{lllllll}
\hline & & PSAP & FIFO & XYY & ETFD & SGFD \\
\hline \multirow{2}{*}{$\begin{array}{l}\text { Original form (OLS) } \\
\text { Eqs. (2)-(6) }\end{array}$} & RMSE & 8.247 & 7.959 & 8.127 & 8.631 & 9.490 \\
& AE-10 & $70.8 \%$ & $72.2 \%$ & $72.2 \%$ & $68.1 \%$ & $55.6 \%$ \\
& AE-20 & $93.1 \%$ & $94.4 \%$ & $94.4 \%$ & $90.3 \%$ & $83.3 \%$ \\
\hline \multirow{2}{*}{ New form (OLS) } & RMSE & 8.125 & 8.032 & 8.159 & 8.484 & - \\
Eqs. (8)-(11) & AE-10 & $75.0 \%$ & $66.7 \%$ & $65.3 \%$ & $69.4 \%$ & - \\
\hline \multirow{2}{*}{$\begin{array}{l}\text { Original form } \\
\text { (WSL) }\end{array}$} & AE-20 & $95.8 \%$ & $91.7 \%$ & $93.1 \%$ & $91.7 \%$ & - \\
\hline \multirow{2}{*}{$\begin{array}{l}\text { Nonlinear effect } \\
\text { (OLS) }\end{array}$} & AE-10 & $72.2 \%$ & $73.6 \%$ & $70.8 \%$ & $69.4 \%$ & $55.6 \%$ \\
& RMSE & 8.096 & 7.942 & 8.102 & 8.180 & - \\
\hline \multirow{2}{*}{ Time-varying } & AE-10 & $68.1 \%$ & $68.1 \%$ & $69.4 \%$ & $65.3 \%$ & - \\
(original form) & AE-20 & $95.8 \%$ & $94.4 \%$ & $95.8 \%$ & $93.1 \%$ & - \\
\hline \multirow{2}{*}{ Time-varying } & RMSE & 8.331 & 7.984 & 8.188 & 8.664 & 8.953 \\
(new form) & AE-10 & $70.8 \%$ & $73.6 \%$ & $70.8 \%$ & $69.4 \%$ & $59.7 \%$ \\
\hline \multirow{2}{*}{ User learning } & AE-20 & $93.1 \%$ & $94.4 \%$ & $94.4 \%$ & $88.9 \%$ & $87.5 \%$ \\
\hline & RMSE & 8.017 & 7.770 & 7.948 & 8.446 & - \\
\hline & AE-10 & $75.0 \%$ & $72.2 \%$ & $75.0 \%$ & $72.2 \%$ & - \\
\hline & AE-20 & $94.4 \%$ & $97.2 \%$ & $97.2 \%$ & $91.7 \%$ & - \\
\hline
\end{tabular}

\subsection{Rational behavior adjustment process}

The evolution processes in Eqs. (2)-(6), as well as NTP and PDS, have commonality that the total travel cost of the network would decreases based on the previous day's path travel costs, i.e. $\left(f^{(n+1)}-f^{(n)}\right) \cdot c^{(n)}<0$, until DUE is reached. This property was noticed by Zhang et al. (2001) and Yang and Zhang (2009), and was named as the "rational behavior adjustment process" (RBAP) by the latter. Guo et al. $(2013,2015)$ pointed out that the same feature can be found in the link-based models such as He et al. (2010), Han and Du (2012) and Smith and Mounce (2011). The RBAP-like models with elastic demand were proposed in Sandholm (2002, 2005), Yang (2007) and Li et al. (2012).

To verify the RBAP property, we plot $\left(f^{(n+1)}-f^{(n)}\right) \cdot c^{(n)}$ against $n$ in Figure 6: the maximum value is 510, and the minimum is -14072 . Among the 25 points, only 8 of them (marked as " $x ")$ are nonnegative. Therefore, the assumption of RBAP is well satisfied in our experiment. An additional observation is that the absolute value of $\left(f^{(n+1)}-f^{(n)}\right) \cdot c^{(n)}$ was gradually shrinking to zero along the day-to-day process. 


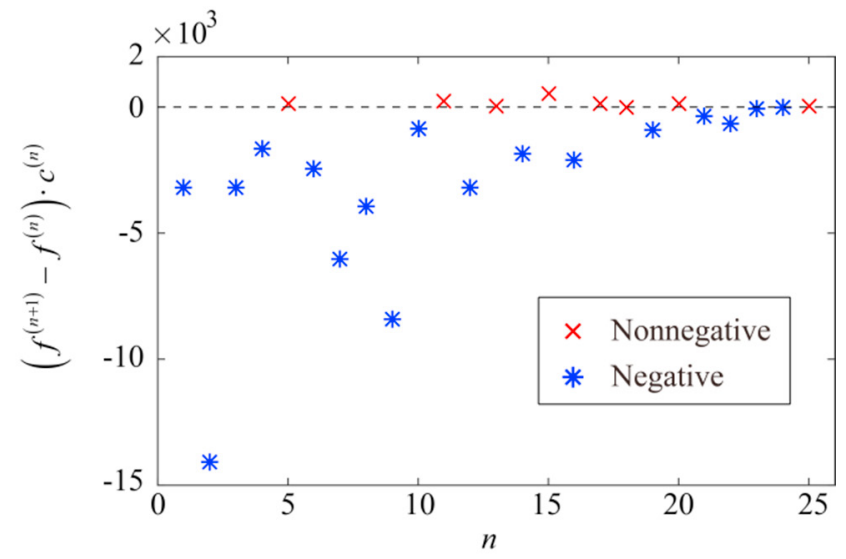

Figure 6. Verification of RBAP.

\subsection{Perspective of Lyapunov function}

Lyapunov's second theorem is widely used in the literature for proving the stability and convergence of a day-to-day model, with reliance on strictly decreasing Lyapunov functions. As pointed out by Xiao et al. (2016), the Lyapunov functions can represent the energies of the transportation networks, and conversely, energies of the transportation networks, once defined, can be used as the Lyapunov functions to investigate the stability of a day-to-day model. In this subsection, we examine the evolution of different Lyapunov functions previously used in the literature.

The most widely-used Lyapunov function is the Beckmann's transformation given as

$$
V^{(n)}=\sum_{a \in A} \int_{0}^{v_{a}^{(n)}} t_{a}(\omega) \mathrm{d} \omega-\sum_{a \in A} \int_{0}^{v_{a}^{*}} t_{a}(\omega) \mathrm{d} \omega
$$

where $v_{a}^{*}$ is the DUE flow on link $a \in A$, as in Guo et al. (2013), Han and Du (2012), Jin (2007), Peeta and Yang (2003) and Smith and Mounce (2011). Other forms of Lyapunov function include the following one in Smith (1984)

$$
V^{(n)}=\sum_{r \in R} \sum_{s \in R} f_{r}^{(n)}\left[c_{r}^{(n)}-c_{s}^{(n)}\right]_{+}^{2}
$$

and the Euclidean distance between the non-DUE and DUE path flows (Nagurney and Zhang, 1997) or link flows (Guo et al., 2015), i.e.,

$$
\begin{aligned}
& V^{(n)}=\sum_{a \in A}\left(v_{a}^{(n)}-v_{a}^{*}\right)^{2} \\
& V^{(n)}=\sum_{r \in R}\left(f_{r}^{(n)}-f_{r}^{*}\right)^{2}
\end{aligned}
$$

where $f_{r}^{*}$ is the DUE flow on path $r \in R$. Notably, the original forms of all these four Lyapunov functions are used for continuous-time day-to-day models. When being used for the discrete-time models here, they may not be strictly decreasing anymore. To compare these Lyapunov functions, we plot $V^{(n)} / \max _{n} V^{(n)}$ against $n$ in Figure 7. The evolution of the four different forms in Eqs. (32)-(35) is quite similar, which all gradually approach to zero with obvious fluctuation. An unexpected peak appears on the $9^{\text {th }}$ day, consistent with the peak of flow on Path 2 on the same day (see Figure 2), which is mainly caused by the massive flow switch from Paths 1 and 3 to Path 2 without explicable reasons. 


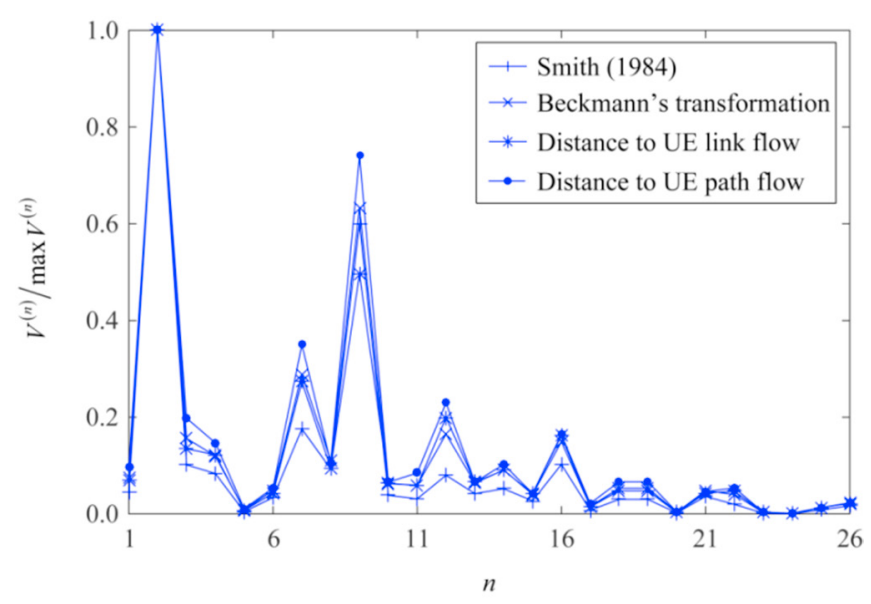

Figure 7. Evolution of the Lyapunov functions.

\section{Conclusions and future research directions}

In this paper, we adopted regression analysis to investigate the existing day-to-day models based on a virtual route choice experiment. We identified some issues (such as heterogeneity and autocorrelation) to which attentions should be paid in such qualitative research; we also observed some interesting properties of the day-to-day model that can be considered in both empirical and theoretical research. The heterogeneity and autocorrelation found in regression could be a hint for missing features in a day-to-day model. Modifying the model form can help tackle the heterogeneity and autocorrelation; however, it may deprive the key mathematical properties of a day-to-day model including steady states and stability. Alternatively, we adopted the WLS method that keeps the model forms intact. Various assumptions on participants' route choice behaviors are examined. The findings include:

(1) It is suggested that the influence of path cost difference on route swapping rate is increasing and concave in PSAP, FIFO, XYY and ETFD; however, the influence of path flow varies.

(2) The path preference was not detected, which might be due to the experiment setting. The participants are encouraged to achieve the minimum travel time, so they have no reason to prefer one path to the other.

(3) It is found that the parameter is time-varying in SGFD but not in PSAP, FIFO, XYY or EGFD, while an altered form of FIFO showed a time-varying parameter. This implies that the time-varying property of the parameter might be model-dependent.

(4) The learning-like behavior was found only in SGFD, which is consistent with the autocorrelation found in the original SGFD form. Such observation links the autocorrelation in an econometric analysis with the cognitive assumption in decision-making. The failure to detect the learning behavior in most models may attribute to the fact that we did not explicitly provide the historical travel times when the participants were making choices, or that their cognitive behaviors during the decision-making are much more complex than the simple learning process assumed.

(5) The comparison between ETFD and SGFD illustrated how the modification of model forms can lead to distinct conclusions on time-varying parameter and learning behavior.

(6) PSAP, FIFO and XYY showed similar prediction power with acceptable accuracy for the path flow prediction, while ETFD and SGFD are falling not too far behind.

(7) The assumption of rational behavior adjustment process is well satisfied.

(8) The four Lyapunov functions used in the literature for stability analysis evolve similarly and exhibit the same 
tendency to approaching zero.

Among the findings are some unsolved questions, such as the assumptions of participants' path preference and learning behavior, as well as whether and how the flows will affect the flow swapping, although intuitively they should be related to each other. Answering these questions requires richer data either from the virtual experiments under more practical settings or from real urban road networks.

Due to the experiment settings, we are only able to examine the DUE-based day-to-day models. For future research, we are also interested in investigating those day-to-day models based on broader behavioral settings, such as stochastic UE and boundedly rational UE. Also, it is good to explore via the virtual experiments, how the day-to-day models perform for modelling the scenarios under traffic disruption or with the provision of traffic information. The demand can be well fixed in a laboratory experiment; however, in the real world the demand is always varying. Thus, another interesting extension is to consider a varying-demand environment, which could be either elastic with respect to cost and/or varying with departure time. Interestingly, there are less elastic-demand day-to-day models rather than fixed-demand models in the literature; therefore, the empirical studies may help enrich the elastic-demand models in a bottom-up manner. Moreover, incorporating departure time choice (Mahmassani, 1990; Mahmassani et al., 1986; Xiao and Lo, 2016) would add another dimension together with the route choices considered in a general network context.

We are also enlightened by another idea of revealing individual-level characteristics by investigating aggregate-level models. It would be particularly interesting to develop a methodology for incorporating individual heterogeneity in a model based on aggregate-level observations (such as flows), and detecting such heterogeneity from empirical data. Also, as some day-to-day models are built upon the assumption of travelers' perceiving behavior on travel times, and such perception is generally difficult to measure, it would be valuable to figure out a way to calibrate such models based on measurable variables such as flows and travel times. Finally yet importantly, quantitative analysis at the individual level, being the conventional way of studying the route changing behavior, could provide valuable information for the analysis at a macroscopic level as we did. Unlike the above-mentioned idea of detecting individual-level heterogeneity in an aggregate model, the microscopic analysis could conversely provide reference for the macroscopic level analysis.

\section{Acknowledgement}

The authors wish to express their thanks to Hani Mahmassani and two anonymous reviewers for their valuable comments on the earlier version of the paper. The work described in this paper was jointly supported by grants from the National Natural Science Foundation of China $(71622007,71201135,71371020)$ and the Research Grants Council of the Hong Kong SAR of China (HKUST16211114).

\section{References}

Avineri, E., Prashker, J., 2006. The impact of travel time information on travelers' learning under uncertainty. Transportation 33 (4), $393-408$. Avineri, E., Prashker, J.N., 2005. Sensitivity to travel time variability: travelers' learning perspective. Transportation Research Part C 13 (2), 157183.

Bar-Yosef, A., Martens, K., Benenson, I., 2013. A model of the vicious cycle of a bus line. Transportation Research Part B 54, 37-50.

Ben-Elia, E., Di Pace, R., Bifulco, G.N., Shiftan, Y., 2013. The impact of travel information's accuracy on route-choice. Transportation Research Part C 26, 146-159.

Ben-Elia, E., Erev, I., Shiftan, Y., 2008. The combined effect of information and experience on drivers' route-choice behavior. Transportation 35 (2), 165-177. 
Bie, J., Lo, H.K., 2010. Stability and attraction domains of traffic equilibria in a day-to-day dynamical system formulation. Transportation Research Part B 44 (1), 90-107.

Bifulco, G.N., Cantarella, G.E., Simonelli, F., Velonà, P., 2016. Advanced traveller information systems under recurrent traffic conditions: Network equilibrium and stability. Transportation Research Part B 92, 73-87.

Cantarella, G.E., 2013. Day-to-day dynamic models for Intelligent Transportation Systems design and appraisal. Transportation Research Part C 29, 117-130.

Cantarella, G.E., Cascetta, E., 1995. Dynamic processes and equilibrium in transportation networks: Towards a unifying theory. Transportation Science 29 (4), 305-329.

Cantarella, G.E., Velonà, P., Vitetta, A., 2012. Signal setting with demand assignment: global optimization with day-to-day dynamic stability constraints. Journal of Advanced Transportation 46 (3), 254-268.

Cantarella, G.E., Velonà, P., Watling, D., 2015. Day-to-day dynamics \& equilibrium stability in a two-mode transport system with responsive bus operator strategies. Networks and Spatial Economics 15 (3), 485-506.

Cascetta, E., 1989. A stochastic process approach to the analysis of temporal dynamics in transportation networks. Transportation Research Part B 23 (1), 1-17.

Cascetta, E., Cantarella, G.E., 1991. A day-to-day and within-day dynamic stochastic assignment model. Transportation Research Part A 25 (5), 277-291.

Cascetta, E., Cantarella, G.E., 1993. Modelling dynamics in transportation networks: State of the art and future developments. Simulation Practice and Theory $1(2), 65-91$

Cho, H., Hwang, M., 2005. Day-to-day vehicular flow dynamics in intelligent transportation network. Mathematical and Computer Modelling 41 (4-5), 501-522.

Chow, G.C., 1960. Tests of equality between sets of coefficients in two linear regressions. Econometrica 28 (3), 591-605.

Dechenaux, E., Mago, S., Razzolini, L., 2014. Traffic congestion: an experimental study of the Downs-Thomson paradox. Experimental Economics 17 (3), 461-487.

Davis, G.A., Nihan, N.L., 1993. Large population approximations of a general stochastic traffic assignment model. Operations Research 41 (1), 169-178.

Di, X., Liu, H.X., Ban, X., Yu, J., 2015. Submission to the DTA 2012 special issue: On the stability of a boundedly rational day-to-day dynamic. Networks and Spatial Economics 15 (3), 537-557.

Farokhi, F., Johansson, K.H., 2015. A piecewise-constant congestion taxing policy for repeated routing games. Transportation Research Part B 78, 123-143.

Friesz, T.L., Bernstein, D., Kydes, N., 2004. Dynamic Congestion Pricing in Disequilibrium. Networks and Spatial Economics 4 (2), 181-202.

Friesz, T.L., Bernstein, D., Mehta, N.J., Tobin, R.L., Ganjalizadeh, S., 1994. Day-to-day dynamic network disequilibria and idealized traveler information systems. Operations Research 42 (6), 1120-1136.

Guo, R.Y., Yang, H., Huang, H.J., 2013. A discrete rational adjustment process of link flows in traffic networks. Transportation Research Part C 34, 121-137.

Guo, R., Yang, H., Huang, H., Tan, Z., 2015. Link-based day-to-day network traffic dynamics and equilibria. Transportation Research Part B 71, 248-260.

Guo, R., Yang, H., Huang, H., Tan, Z., 2016. Day-to-day flow dynamics and congestion control. Transportation Science 50 (3), $982-997$.

Guo, X., 2013. Toll sequence operation to realize target flow pattern under bounded rationality. Transportation Research Part B 56, $203-216$.

Guo, X., Liu, H.X., 2011. Bounded rationality and irreversible network change. Transportation Research Part B 45 (10), 1606-1618.

Han, L., Du, L., 2012. On a link-based day-to-day traffic assignment model. Transportation Research Part B 46 (1), $72-84$.

Hazelton, M.L., 2002. Day-to-day variation in Markovian traffic assignment models. Transportation Research Part B 36 (7), $637-648$.

Hazelton, M.L., Parry, K., 2016. Statistical methods for comparison of day-to-day traffic models. Transportation Research Part B 92, $22-34$.

Hazelton, M.L., Watling, D.P., 2004. Computation of equilibrium distributions of Markov traffic-assignment models. Transportation Science 38 (3), 331-342.

He, X., Guo, X., Liu, H.X., 2010. A link-based day-to-day traffic assignment model. Transportation Research Part B 44 (4), $597-608$.

He, X., Liu, H. X., 2012. Modeling the day-to-day traffic evolution process after an unexpected network disruption. Transportation Research Part B 46 (1), 50-71.

Horowitz, J.L., 1984. The stability of stochastic equilibrium in a two-link transportation network. Transportation Research Part B 18 (1), $13-28$.

Hu, T.Y., Mahmassani, H.S., 1997. Day-to-day evolution of network flows under real-time information and reactive signal control. Transportation Research Part C 5 (1), 51-69.

Huang, W., Viti, F., Tampère, C.M.J., 2016. Repeated anticipatory network traffic control using iterative optimization accounting for model bias correction. Transportation Research Part C 67, 243-265.

Jin, W., 2007. A dynamical system model of the traffic assignment problem. Transportation Research Part B 41 (1), 32-48.

Li, X., Yang, H., 2016. Dynamics of modal choice of heterogeneous travelers with responsive transit services. Transportation Research Part C 68, 333-349 
Li, Y., Tan, Z., Chen, Q., 2012. Dynamics of a transportation network model with homogeneous and heterogeneous users. Discrete Dynamics in Nature and Society 2012, 1-16.

Liu, R., Smith, M., 2015. Route choice and traffic signal control: A study of the stability and instability of a new dynamical model of route choice and traffic signal control. Transportation Research Part B 77, 123-145.

Lotan, T., 1997. Effects of familiarity on route choice behavior in the presence of information. Transportation Research Part C 5 (3-4), $225-243$.

Lu, X., Gao, S., Ben-Elia, E., 2011. Information impacts on route choice and learning behavior in a congested network. Transportation Research Record 2243, 89-98.

Mahmassani, H.S., 1990. Dynamic models of commuter behavior: Experimental investigation and application to the analysis of planned traffic disruptions. Transportation Research Part A 24 (6), 465-484.

Mahmassani, H.S., Chang, G.L., 1986. Experiments with departure time choice dynamics of urban commuters. Transportation Research Part B 20 (4), 297-320.

Mahmassani, H.S., Chang, G., 1987. On boundedly rational user equilibrium in transportation systems. Transportation Science 21 (2), 89-99.

Mahmassani, H.S., Chang, G.L., Herman, R., 1986. Individual decisions and collective effects in a simulated traffic system. Transportation Science 20 (4), 258-271.

Mahmassani, H.S., Jou, R.C., 2000. Transferring insights into commuter behavior dynamics from laboratory experiments to field surveys. Transportation Research Part A 34 (4), 243-260.

Mahmassani, H.S., Herman, R., 1990. Interactive experiments for the study of tripmaker behaviour dynamics in congested commuting systems. In: Jones, P. (Ed.) Developments in Dynamic and Activity-Based Approaches to Travel Analysis. Avebury, Gower, Aldershot, England, pp. 272-298.

Mahmassani, H.S., Stephan, D.G., 1988. Experimental investigation of route and departure time choice dynamics of urban commuters. Transportation Research Record 1203, 69-84.

Meneguzzer, C., Olivieri, A., 2013. Day-to-day traffic dynamics: laboratory-like experiment on route choice and route switching in a simple network with limited feedback information. Procedia - Social and Behavioral Sciences 87, 44-59.

Morgan, J., Orzen, H., Sefton, M., 2009. Network architecture and traffic flows: Experiments on the Pigou-Knight-Downs and Braess Paradoxes. Games and Economic Behavior 66 (1), 348-372.

Mounce, R., Carey, M., 2011. Route swapping in dynamic traffic networks. Transportation Research Part B 45 (1), $102-111$.

Nagurney, A., Zhang, D., 1997. Projected dynamical systems in the formulation, stability analysis, and computation of fixed-demand traffic network equilibria. Transportation Science 31 (2), 147-158.

Parry, K., Hazelton, M.L., 2013. Bayesian inference for day-to-day dynamic traffic models. Transportation Research Part B 50, $104-115$.

Rapoport, A., Gisches, E.J., Daniel, T., Lindsey, R., 2014. Pre-trip information and route-choice decisions with stochastic travel conditions: Experiment. Transportation Research Part B 68, 154-172.

Rapoport, A., Kugler, T., Dugar, S., Gisches, E.J., 2009. Choice of routes in congested traffic networks: Experimental tests of the Braess Paradox. Games and Economic Behavior 65 (2), 538-571.

Sandholm, W.H., 2002. Evolutionary implementation and congestion pricing. The Review of Economic Studies 69 (3), $667-689$.

Sandholm, W.H., 2005. Excess payoff dynamics and other well-behaved evolutionary dynamics. Journal of Economic Theory 124 (2), $149-170$.

Smith, M.J., 1983. The existence and calculation of traffic equilibria. Transportation Research Part B 17 (4), 291-303.

Smith, M.J., 1984. The stability of a dynamic model of traffic assignment - an application of a method of Lyapunov. Transportation Science 18 (3), 245-252.

Smith, M.J., Liu, R., Mounce, R., 2015. Traffic control and route choice: Capacity maximisation and stability. Transportation Research Part B 81, 863-885.

Smith, M.J., Mounce, R., 2011. A splitting rate model of traffic re-routing and traffic control. Transportation Research Part B 45 (9), $1389-1409$.

Smith, M.J., Watling, D.P., 2016. A route-swapping dynamical system and Lyapunov function for stochastic user equilibrium. Transportation Research Part B 85, 132-141.

Srinivasan, K.K., Mahmassani, H.S., 2003. Analyzing heterogeneity and unobserved structural effects in route-switching behavior under ATIS: a dynamic kernel logit formulation. Transportation Research Part B 37 (9), 793-814.

Tan, Z., Yang, H., Guo, R., 2015. Dynamic congestion pricing with day-to-day flow evolution and user heterogeneity. Transportation Research Part C 61, 87-105.

Wang, Y., Liu, H., Han, K., Friesz, T.L., Yao, T., 2015. Day-to-day congestion pricing and network resilience. Transportmetrica A 11 (9), 873895.

Watling, D., 1999. Stability of the stochastic equilibrium assignment problem: A dynamical systems approach. Transportation Research Part B 33 (4), 281-312.

Watling, D.P., Cantarella, G.E., 2015. Model representation \& decision-making in an ever-changing world: The role of stochastic process models of transportation systems. Networks and Spatial Economics 15 (3), 843-882.

Wu, J., Sun, H., Wang, D.Z.W., Zhong, M., Han, L., Gao, Z., 2013. Bounded-rationality based day-to-day evolution model for travel behavior analysis of urban railway network. Transportation Research Part C 31, 73-82. 
Xiao, F., Yang, H., Ye, H., 2016. Physics of day-to-day network flow dynamics. Transportation Research Part B 86, 86-103.

Xiao, L., Lo, H., 2015. Combined route choice and adaptive traffic control in a day-to-day dynamical system. Networks and Spatial Economics $15(3), 697-717$.

Xiao, Y., Lo, H.K., 2016. Day-to-day departure time modeling under social network influence. Transportation Research Part B 92, 54-72.

$\mathrm{Xu}$, M., Meng, Q., Huang, Z., 2016. Global convergence of the trial-and-error method for the traffic-restraint congestion-pricing scheme with day-to-day flow dynamics. Transportation Research Part C 69, 276-290.

Yang, F., 2005. An Evolutionary Game Theory Approach to the Day-to-Day Traffic Dynamics. Dissertation, University of Wisconsin-Madison.

Yang, F., 2007. Day-to-day dynamic optimal tolls with elastic demand, working paper.

Yang, F., Szeto, W.Y., 2006. Day-to-day dynamic congestion pricing policies towards system optimal. Proceedings of the First International Symposium on Dynamic Traffic Assignment, Leeds, United Kingdom, 266-275.

Yang, F., Yin, Y., Lu, J., 2007. Steepest descent day-to-day dynamic toll. Transportation Research Record 2039, 83-90.

Yang, F., Zhang, D., 2009. Day-to-day stationary link flow pattern. Transportation Research Part B 43 (1), 119-126.

Yang, H., Kitamura, R., Jovanis, P.P., Vaughn, K.M., Abdel-Aty, M.A., 1993. Exploration of route choice behavior with advanced traveler information using neural network concepts. Transportation 20 (2), 199-223.

Ye, H., Yang, H., 2013. Continuous price and flow dynamics of tradable mobility credits. Transportation Research Part B 57, 436-450.

Ye, H., Yang, H., 2016. Rational behavior adjustment process with boundedly rational user equilibrium. Transportation Science (accepted)

Ye, H., Yang, H., Tan, Z., 2015. Learning marginal-cost pricing via a trial-and-error procedure with day-to-day flow dynamics. Transportation Research Part B 81, 794-807.

Zhang, D., Nagurney, A., 1996. On the local and global stability of a travel route choice adjustment process. Transportation Research Part B 30 (4), 245-262.

Zhang, D., Nagurney, A., Wu, J., 2001. On the equivalence between stationary link flow patterns and traffic network equilibria. Transportation Research Part B 35 (8), 731-748. 